\title{
Body weight reduction prior to quinine adulteration of water: Interactive complexities in measures of ingestive behavior
}

\author{
P. J. WATSON, MARTHA L. SWINDOLL, and MICHAEL D. BIDERMAN \\ University of Tennessee, Chattanooga, Tennessee 37402
}

\begin{abstract}
Rats were presented with $.1 \%(w / v)$ quinine-adulterated water after they had been predeprived of food down to $70 \%$ of their ad lib body weight (PD-Q group) or after no predeprivation experience (ND-Q group). Both groups initially reduced their food intake and lost weight. Following adaptation to the adulterated fluid, PD-Q animals displayed a relatively higher rate of weight consolidation; however, these subjects eventually stabilized at a weight level below that of the ND-Q rats. These data (1) demonstrate at least some specificity to the predeprivation effects observed with lateral hypothalamic (LH) lesions, (2) suggest the probable independence of predeprivation and $\mathrm{LH}$ adipsia effects during intervals immediately after surgery, and (3) document the interactive weight effects produced by food predeprivation and fluid adulteration.
\end{abstract}

One suggested outcome of lateral hypothalamic (LH) lesions is body weight set point reduction (Powley \& Keesey, 1970). Support for this position arises from the observation that lesioned rats over long periods of time display a lowered weight level that remains stable relative to controls, and this relatively reduced set point is defended against numerous procedural manipulations of dietary factors (Keesey, Boyle, Kemnitz, \& Mitchel, 1976). In addition, elimination of postlesion aphagia is possible through presurgical lowering of body weight to a level below the lesion-induced set point, and these predeprived animals display an immediate weight gain instead of a weight loss (Powley \& Keesey, 1970).

Research has recently suggested that some lesioninduced alterations may arise secondarily from other symptoms. With LH destruction, for example, a Gordian knot of changes is observed, in which lowered body weights theoretically can be influenced by at least some of the other broad-ranging response alterations produced by the lesion. When multiple behavioral effects follow brain damage, a methodical unraveling of this knot apparently will be necessary to clearly define the factors underlying the syndrome, and demonstration or elimination of potential symptom interaction effects may be equally important in eventually understanding the critical causal processes (Watson, Biderman, \& Warfield, 1981).

A possible challenge to the body weight set point analysis of LH lesion effects involves a focusing on the

Completion of this project was made possible in part by funds obtained from the University of Chattanooga Foundation. Reprint requests should be addressed to P. J. Watson. profound water intake deficits that are a part of the $\mathrm{LH}$ syndrome (Epstein, 1971). Body weight is determined in part by the amount of fluid that an animal is able to ingest (Collier, 1964), and the lowered weights of LH subjects could result secondarily from a disruption of drinking behavior. Although some early data indicated the contribution of a water deficit to the set point effects (Mufson \& Wampler, 1972), other findings subsequently questioned this demonstration (Boyle \& Keesey, 1975). Nevertheless, lesioned animals display at least some increase in food intake and body weight following intravenous infusions of water and isotonic saline (Rowland, 1977), and neurologically intact rats respond to quinine adulteration of their water supply with lowered body weights that resemble those of LH subjects in their stability (Nicolaidis \& Rowland, 1975; see their Figure 1). Rats presented with quinine-adulterated water are also like lesioned animals (Mitchel \& Keesey, 1974) in that reduction in fat stores appears to represent the principal component of the weight loss (Peck, 1978). Other parallels between quinine drinkers and LH subjects involving water ingestion have been documented (Nicolaidis \& Rowland, 1975; Rowland \& Flamm, 1977), and the need to explore additional analogues between quinine drinkers and lesioned animals has been mentioned (Nicolaidis \& Rowland, 1975). The purpose of this study was to examine the effects of body weight reduction on the reactions of rats to adulterated water. Prolonged food deprivation results in absolute body fluid depletion (Kutscher, 1971), and predeprived animals may be less debilitated after lesions, in part because their hydrational state more closely approximates the eventual lesion-induced state. 


\section{METHOD}

\section{Animals}

Experimentally naive, adult male Long-Evans hooded rats served as subjects. These 22 animals weighed $404-540 \mathrm{~g}$ at the beginning of the study and were obtained from the departmental colony.

\section{Procedure}

Starting on Day 1 , subjects were individually housed in $7 \times 7 \times 9.5$ in. stainless steel cages. Room temperature was set at $68^{\circ} \mathrm{F}$, and lighting was turned on at $0800 \mathrm{~h}$ and turned off at $2000 \mathrm{~h}$. All animals had been kept in group cages but were adapted to the individual cages for 7 days prior to implementation of any procedures. Unless otherwise specified, water and Purina Lab Chow pellets were made available ad lib.

Three groups of animals were formed: the predeprivation/ quinine group ( $P D-Q: N=8$ ), the no-deprivation/quinine group (ND-Q: $N=8$ ), and the no-deprivation/no-quinine control group (C: $N=6)$. Group assignment was made in a quasi-random manner that ensured relatively equal initial body weights across the three groups. Following the 7-day adaptation period, PD-Q rats were placed on a food restriction schedule involving 8-10 $\mathrm{g}$ Lab Chow/day, presented during the light phase of the diurnal cycle. Once an animal reached $70 \%$ of its initial body weight, it received varying amounts of food so that its weight was maintained near $70 \%$ until all PD-Q subjects had reached this criterion, an event that occurred on Day 38 . Use of this $70 \%$ value represented an extreme precaution designed to insure that body weight percentages were clearly reduced below the lowest percentages caused by water adulteration alone. At no time during the study did ND-Q rats display reductions below $97 \%$ of their initial weight. In addition, it should be noted that care was taken to guarantee gradual fasting of PD-Q subjects down to the target percentage (Grijalva, Lindholm, Schallert, \& Bicknell, 1976).

Next, PD-Q and ND-Q subjects were given access to a $.1 \%$ (w/v) solution of quinine hydrochloride (Sigma Co.) instead of water. Controls continued to receive water and all animals had ad-lib access to food. These procedures were continued for 34 days, with daily body weight, food consumption, and fluid intake monitored. Food consumption was measured to the nearest $.1 \mathrm{~g}$, and care was taken to collect any large pieces of Lab Chow that had fallen through the cage floor. Fluids were contained in a graduated cylinder held to the front of the cage by a spring, and the volume drunk was recorded to the nearest milliliter. A small dish was placed under the cage, below the drinking spout, to help collect any spillage, and use of the dish seemed successful, except in the very early days of the adulteration procedures. Some of the animals responded to the bitter fluids by vigorously biting at the drinking tube and bottle, and, as a consequence, large amounts of fluid were spilled and could not be collected. The first days of adulterated water intake, therefore, were more variable and slightly elevated through this artifact.

\section{RESULTS}

As in previous investigations (Nicolaidis \& Rowland, 1975), not all animals tolerated the quinine solutions. One PD-Q and three ND-Q rats displayed body weights, fluid intakes, and general behavior activity that suggested that they would die if procedures were continued, and they were dropped from the study. This difference in group acceptance of the fluid was not statistically significant ( $p>.05$, Fisher's exact test). In addition, one PD-Q animal became ill in the middle of the experiment and eventually died, and his data were not included. Final results, therefore, reflected the ingestive behavior of six PD-Q, five ND-Q, and six control subjects.

Figure 1 reviews the body weight findings across all 34 days of fluid adulteration, and Day 0 represents the average body weight on the day prior to initiation of the adulteration procedures. Perhaps the most obvious aspect of the raw data is the similar initial reactions of the PD-Q and ND-Q groups to the adulterated water. Body weights of both groups immediately fell, and the gradual "recovery" process followed a similar pattern until Day 5 . The overall significant group body weight differences $[F(2,14)=32.34, p<.001]$ arose largely from the deprivation-induced weight loss of PD-Q subjects. The change in weight across days was also significant $[F(33,462)=62.15, p<.001]$, as was the Group by Days interaction $[F(66,462)=18.08, p<.001]$. This interaction effect revealed in part the different weight changes of the two quinine groups and of controls during the initial adulteration experience. Further illustration of this effect was possible by comparing weights on the day prior to adulteration with Day 1 weights, and a significant reduction was found for PD-Q $[\mathrm{t}(5)=6.324, \mathrm{p}<.001]$ and ND-Q $[\mathrm{t}(4)=12.97$, $\mathrm{p}<.001]$ animals. In contrast, controls remained essentially unchanged $[\mathrm{t}(5)=-.076, \mathrm{p}>.05]$.

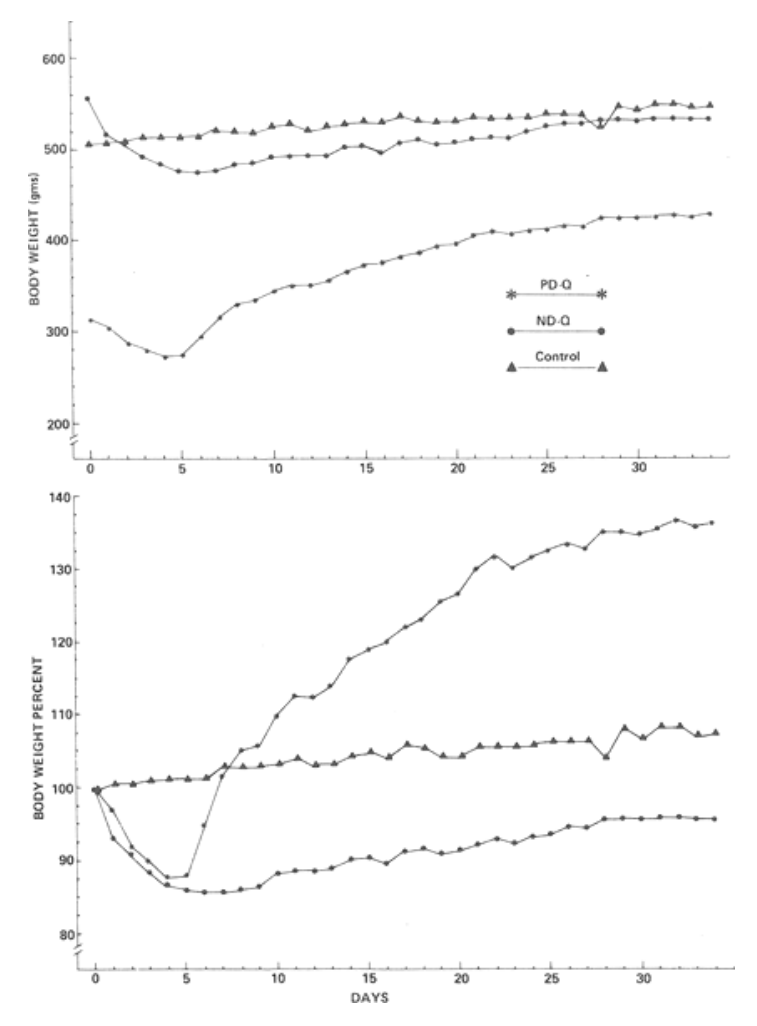

Figure 1. Mean body weight and mean body weight expressed as a percentage of weight on the day prior to adulteration (Day 0). Data are for rats predeprived of food prior to adulteration (PD-Q), for rats not predeprived prior to adulteration (ND-Q), and for controls. 
Day-by-day post hoc analyses were conducted by employing Duncan's range test, with $\mathrm{p}$ set at .05 , and the results indicated that throughout the experiment, PD-Q rats had significantly lower weights than the other two groups, which, in turn, never differed from each other. The three ND-Q rats that were initially dropped from the study had body weights in the bottom half of the group distribution, and the absence of their data resulted in an elevation of the ND-Q group mean relative to controls. Body weights of controls and of the remaining ND-Q subjects nevertheless were not significantly different on the day prior to adulteration $[t(9)=1.76$, $\mathrm{p}>.10]$, but loss of the three ND-Q rats worked against the obtaining of significant control/ND-Q weight differences. At the end of the experiment, PD-Q rats averaged $92 \%$ of their weight on the day before initiation of the food deprivation procedures, and this value was significantly lower than the $108 \%$ of ND-Q and the $115 \%$ of control subjects (Duncan's range test, $\mathrm{p}<.05$ ). The ND-Q and control difference was not significant, however.

A more sensitive analysis of body weight changes was conducted by examining daily body weights for each subject, expressed as a percentage of each subject's body weight on the day prior to adulteration, and these data are also presented in Figure 1. During the first 4 days, both quinine groups responded with successive percentage reductions that were significantly more severe for ND-Q rats on Day 1 ( $p<.5$, Duncan's range test). PD-Q animals began a reversal of this weight loss trend 2 days earlier than ND-Q subjects, and their rate of weight percentage gain was clearly more accelerated. The ND-Q percentage data remained significantly below control data on all days except Day 28 and below the PD-Q data from Day 6 onward (all ps $<.05$, Duncan's range test). The PD-Q percentages remained significantly below those of controls until Day 7; and on Day 11, they reached the significantly higher levels that were maintained until the end of the study (all ps $<.05$ ).

Food and fluid intake measures are presented in Figure 2. Significant differences were obtained in the amount of food consumed [groups: $F(2,14)=8.35$, $\mathrm{p}<.01$ ], and intake changes over time [days: $F(33,462)=26.18, p<.001]$ were not parallel across the three groups [Groups by Days: $F(66,462)=11.00$, $p<.001]$. For the first 7 days, PD-Q and ND-Q rats ate less than controls (all ps $<.05$, Duncan's range test), but only ND-Q animals differed on Day 8. On Days 9-32 essentially equal amounts of food were eaten by the three groups, but on Day 33, PD-Q food intake occurred at a level significantly below that of ND-Q rats and below that of both other groups on Day 34. At the end of the study, therefore, PD-Q animals still remained at a significantly lowered body weight level, and their eating behavior suggested that no return was likely within the short term to the levels of the other groups had the experiment continued.

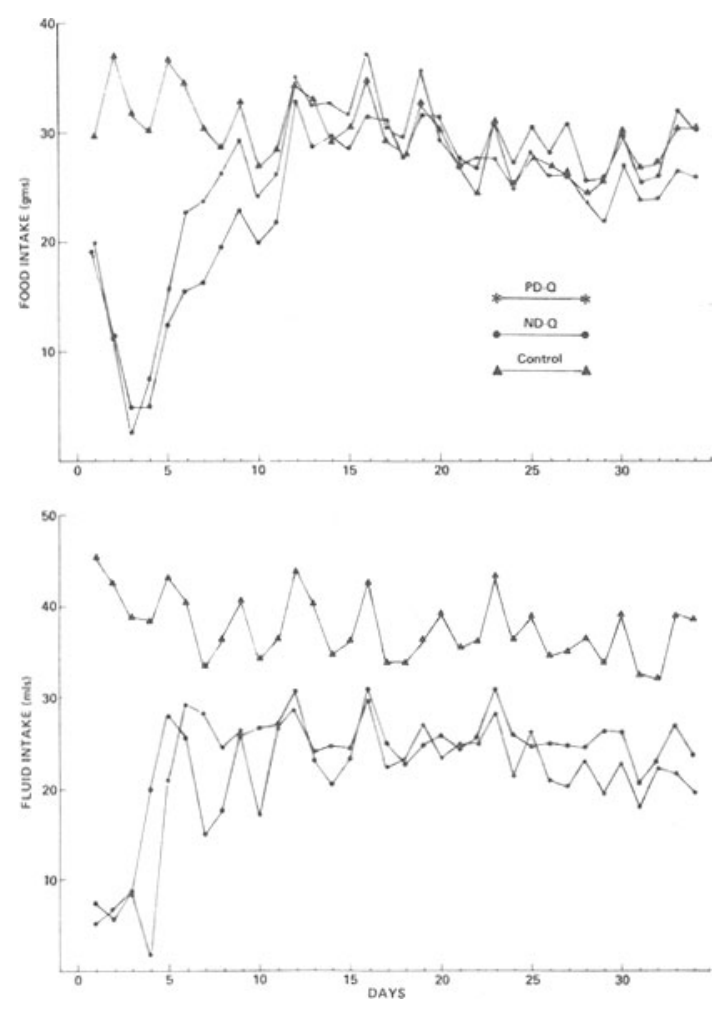

Figure 2. Mean food and mean fluid intake of PD-Q, ND-Q, and control animals.

Group differences in fluid intake were also observed $[\mathrm{F}(2,14)=28.98, \mathrm{p}<.001]$, and the changes over days $[\mathrm{F}(33,462)=9.93, \mathrm{p}<.001]$ interacted with the experimental treatments [Groups by Days: $F(66,462)=$ $4.92, \mathrm{p}<.001]$. Post hoc tests indicated that both quinine groups initially drank less than controls. On Days 4 and 10, PD-Q animals ingested less than ND-Q rats, but a reversal of this relationship was also observed on Day 7 . These initial PD-Q and ND-Q differences should be interpreted with extreme caution because of the previously mentioned measurement problems associated with the aversion reactions of subjects to the quinine. Although the two quinine groups occasionally consumed fluid amounts not significantly different from controls, they nevertheless stabilized at a level that was significantly lower (Days 18-34, all ps < .05).

Fluid/food ratios during the last 10 days averaged .84 for the PD-Q group, .86 for the ND-Q group, and 1.34 for the control group. Ratios for the two quinine groups were essentially equal throughout this interval, but they were reliably below those of controls (all ps $<.05$, Duncan's range test).

\section{DISCUSSION}

Results of this investigation indicate that predeprived animals are able to reduce food intake and body weight in the presence of an appetite-suppressing stimulus like quinine adulteration of 
water. These findings, therefore, demonstrate at least some specificity in the predeprivation effects observed with LH lesions. In other words, the ability of body weight reduction to prevent aphagia and weight loss can be more confidently, although not definitively, related to processes primarily controlling, rather than secondarily influencing, body weight.

The data also reveal the improbability of LH lesion-induced adipsia serving as an important contributor to the initial weight loss effects. If anything, the predeprived animals of this experiment drank more fluids after the food deprivation regimen was removed than did the predeprived LH subjects of other investigations. Nevertheless, PD-Q rats were unlike LH animals in that they were unable to eat enough food to prevent initial weight loss. In fact, their initial food ingestion was not different from that of ND-Q subjects, and their significantly smaller Day 1 weight loss probably reflected a relatively higher absolute level of body fluids available to be lost by the ND-Q animals.

Failure of PD-Q subjects to gain weight at the beginning of adulteration procedures serves to highlight the complex interactions underlying body weight regulation. PD-Q animals were at a weight percentage much below the percentage ever produced by water adulteration alone, and yet they initially lost weight during ad-lib access to food. Nicolaidis and Rowland (1975) have previously reported data making it unlikely that the adulteration-associated weight and food intake reductions were caused by some nonspecific malaise effect of the quinine. In addition, the fact that PD-Q and ND-Q rats stabilized at different weight plateaus supports the idea that experience with reduced food intake may be a sufficient stimulus for altering long-term weight regulation. More compelling demonstrations of this effect have been reported (Sawchenko, Gold, \& Bisson, 1980; Wayner, Stein, Loullis, Barone, Jolicoeur, \& Rondeau, 1978), and they suggest at least some contribution of a lesion-induced acute food intake reduction to the chronic $\mathrm{LH}$ set point effects.

In summary, complex interactions were observed between body weight, food consumption, and fluid ingestion when animals with reduced body weights were presented with a quinineadulterated water supply. The initial failure of these subjects to be hyperphagic and to gain weight makes even more striking the influences of predeprivation on LH-esioned animals.

\section{REFERENCES}

Boyle, P. C., \& Keesey, R. E. Chronically reduced body weight in rats sustaining lesions of the lateral hypothalamus and maintained on palatable diets and drinking solutions. Journal of Comparative and Physiological Psychology, 1975, 88, 218-223.

Collie r, G. Thirst as a determinant of reinforcement. In M. J. Wayner (Ed.), Thirst. London: Pergamon Press, 1964.

EPSTE in, A. N. The lateral hypothalamic syndrome: Its implications for the physiological psychology of hunger and thirst. In
E. Stellar \& J. M. Sprague (Eds.), Progress in physiological psychology (Vol. 4). New York: Academic Press, 1971.

Grijalva, C. V., Lindholm, E., Schallert, T., \& Bicknell, E. Gastric pathology and aphagia following lateral hypothalamic lesions in rats: Effects of preoperative weight reduction. Journal of Comparative and Physiological Psychology, 1976, 90, 505-519.

Keesey, R. E., Boyle, P. C., Kemnitz, J. W., \& Mitchell, J. S. The role of the lateral hypothalamus in determining the body weight set point. In D. Novin, W. Wyrwicka, \& G. Bray (Eds.), Hunger: Basic mechanisms and clinical implications. New York: Raven Press, 1976.

Kutsche R, C. L. Hematocrit, plasma osmolality, and plasma protein concentration as estimators of plasma volume in hooded rats during food and water deprivation. Physiology \& Behavior, 1971, 7, 283-285.

Mitchel, J. S., \& Keesey, R. E. The effects of lateral hypothalamic lesions and castration upon body weight and composition of male rats. Behavioral Biology, 1974, 11, 69-82.

Mufson, E. J., \& WAMPLER, R. S. Weight reduction with palatable food and liquids in rats with lateral hypothalamic lesions. Journal of Comparative and Physiological Psychology, 1972, 80, 382-392.

Nicolaidis, S., \& Rowland, N. Regulatory drinking in rats with permanent access to a bitter fluid source. Physiology \& Behavior, 1975, 14, 819-824.

PECK, J. W. Rats drinking quinine- or caffeine-adulterated water defend lean body weights against caloric and osmotic stress. Physiology \& Behavior, 1978, 21, 599-607.

Powley, T. L., \& KeEsey, R. E. Relationship of body weight to the lateral hypothalamic feeding syndrome. Journal of Comparative and Physiological Psychology, 1970, 70, 25-36.

Rowland, N. Bodyweight following lateral hypothalamic lesions: Set point upset by glucose infusions. Physiology \& Behavior, 1977, 19, 349-353.

Rowland, N., \& Flamm, C. Quinine drinking: More regulatory puzzles. Physiology \& Behavior, 1977, 18, 1165-1170.

Sawchenko, P. E., Gold, R. M., \& Bisson, R. Depriving or pair feeding intact controls induces some persisting deficits similar to those of the recovered lateral rat. Journal of Comparative and Physiological Psychology, 1980, 94, 128-144.

Watson, P. J., Biderman, M. D., \& Warfield, D. R. Eating induced by injections of 2-deoxy-d-glucose in neurologically intact hypodipsic rats. International Journal of Neuroscience, 1981, 15, 95-98.

Wayner, M. J., Stein, J. M., Loullis, C. C., Barone, F. C., Jolicoevr, F. B., \& Rondenu, D. B. Effects of two body weight reduction regimens on schedule dependent and schedule induced behavior. Physiology \& Behavior, 1978, 21, 639-645.

(Received for publication July 9, 1982.) 